\title{
Powering stem cell decisions with ubiquitin
}

\author{
Achim Werner ${ }^{\star, 1}$ and Michael Rape $e^{1,2}$ \\ Cell Death and Differentiation (2017) 24, 1823-1824; doi:10.1038/cdd.2017.142; published online 8 September 2017
}

Many types of pluripotent stem cells exist in a quiescent state within specialized hypoxic niches of the metazoan body. Upon receiving appropriate inputs, these progenitor cells can start to proliferate or commit to differentiation programs that give rise to the different cell types of the developing organism. ${ }^{1}$ Stem cells and their derivatives tailor their metabolic program to the demands given by their respective cellular states: ${ }^{2}$ while stem cells rely on anaerobic glycolytic energy production to account for hypoxic conditions in the niche and to minimize production of toxic reactive oxygen species, differentiating cells switch to aerobic metabolism to increase energy production. To achieve this metabolic switch, stem cells regulate the biogenesis and function of mitochondria, the cell's powerhouses for anaerobic energy production, yet how this process is regulated is not well understood.

In an article in Nature Cell Biology, ${ }^{3}$ Donato et al. reveal a critical function for the ubiquitin-proteasome system in regulating mitochondrial mass expansion during mouse embryonic stem cell (mESC) differentiation. Key target of this pathway is the Kif1-binding protein (KBP) that together with the mitochondrial-associated kinesin KIF1Balpha controls microtubule-mitochondria interactions. The ubiquitindependent degradation of KBP in mESCs limits mitochondrial biogenesis for faithful stem cell maintenance, while inhibition of KBP turnover and its subsequent accumulation at the onset of differentiation allows for expansion of the mitochondrial network for proper differentiation (Figure 1).

The investigators uncovered this pathway by setting out to study the stem cell-specific F-box protein FBXO15, one of $\sim 70$ substrate adaptors for SCF (Skp1-Cul1-F-box) ubiquitin E3 ligase complexes. FBXO15 is preferentially expressed in undifferentiated cells, and indeed, had previously been used as a marker for induced pluripotent stem cells. ${ }^{4}$ Through proteomic, biochemical, and genetic analyses, the authors identified KBP as substrate of $\mathrm{SCF}^{\mathrm{FBXO} 15}$-mediated proteasomal degradation and determined the molecular basis underlying KBP ubiquitylation. While many F-box proteins recognize their targets following substrate phosphorylation, ${ }^{5}$ FBXO15 relies on prior acetylation of a lysine residue within a conserved degron motif in KBP. Substitution of the critical lysine residue with arginine rendered $\mathrm{KBP}$ insensitive to $\mathrm{SCF}^{\mathrm{FBXO15}}$-mediated degradation. Thus, $\mathrm{SCF}^{\mathrm{FBXO15}}$ targets acetylated KBP for proteasomal degradation, rendering
FBX015 one of very few first known acetylation-dependent E3 ligases.

As KBP is turned over in mESCs, but not during differentiation, the acetylation of this important cellular regulator is likely regulated during development. To identify the relevant enzymes mediating KBP acetylation, the authors took a candidate approach. These experiments pointed to the mitochondrial acetyltransferase GCN5L1 and L-threonine dehydrogenase (TDH), an enzyme known to produce mitochondrial acetyl-coA in mESCs, ${ }^{6}$ as factors required for KBP acetylation. Indeed, siRNA-mediated depletion or pharmacological inhibition of GCN5L1 or TDH dampened the acetylation of KBP and consequently stabilized this protein in mESCs. Thus, GCN5L1 and TDH, through mediating the acetylation of $\mathrm{KBP}$, are essential for $\mathrm{SCF}^{\mathrm{FBXO15}}$-dependent turnover of KBP in mESCs (Figure 1).

Previous studies had suggested roles for microtubules in mitochondrial biogenesis ${ }^{7}$ and for the KBP-KIF1Balpha complex in connecting mitochondria to microtubules. ${ }^{8}$ Based on these observations, the authors wondered whether interfering with KBP degradation in mESCs affects microtubule-mitochondria interactions and mitochondrial biogenesis. Confocal microscopy and quantitative image analyses revealed that expression of degradation-resistant KBP resulted in elevated co-localization of mitochondria and tubulin in $\mathrm{mESC}$ and a significant increase in the number and volume of mitochondria per cell. In line with these findings, impeding the proteolytic circuitry by depleting FBXO15, deleting the FBX015 gene, or inhibiting $\mathrm{TDH}$, fueled mitochondrial biogenesis in mESCs. As a consequence, mESCs lacking functional $\mathrm{SCF}^{\mathrm{FBXO15}}$ or expressing stabilized KBP consumed more oxygen, produced more ATP, and generated increased amounts of reactive oxygen species, while showing decreased proliferation rates. These findings indicated that KBP degradation by SCF ${ }^{\mathrm{FBXO15}}$ restricts mitochondrial biogenesis and preserves the fitness of $\mathrm{mESC}$, likely by limiting the production of harmful reactive oxygen species during cellular respiration.

Previous reports had shown that FBXO15 and TDH are transcriptionally silenced at the onset of differentiation. ${ }^{6,9}$ This would allow restriction of KBP acetylation and degradation to the pluripotent state and enable accumulation of KBP during early differentiation when mitochondrial biogenesis is activated. Consistent with this model, the authors found that

\footnotetext{
${ }^{1}$ Department of Molecular and Cell Biology, University of California at Berkeley, Berkeley, CA 94720, USA and ${ }^{2}$ Howard Hughes Medical Institute, University of California at Berkeley, Berkeley, CA 94720, USA

*Corresponding author: A Werner, Department of Molecular and Cell Biology, University of California at Berkeley, Berkeley, CA 94720, USA. Tel: (510) 642-0468; E-mail: a.werner@berkeley.edu
} 
Stem cell state: Limited mitochondrial biogenesis through KBP degradation

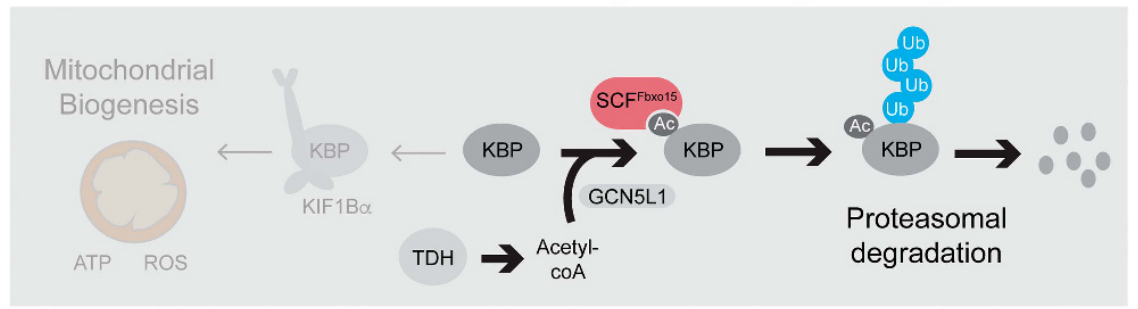

Differentiation: Enhanced mitochondrial biogenesis through stablized KBP

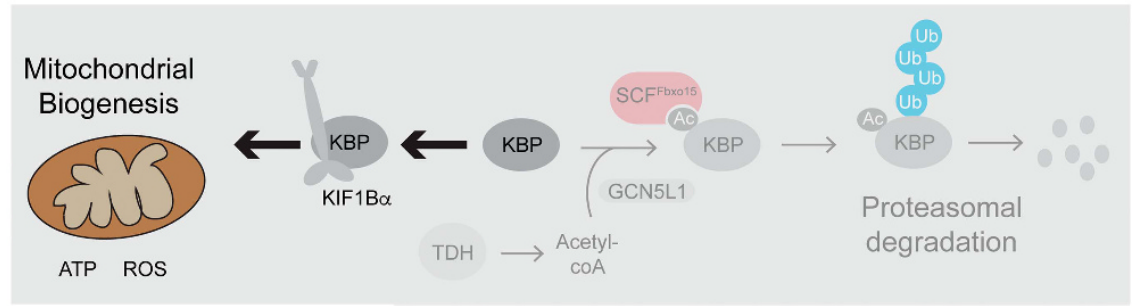

Figure 1 Upper panel: in stem cells, the $\mathrm{E} 3$ ubiquitin ligase $\mathrm{SCF}^{\mathrm{FBX} 15}$ recognizes acetylated $\mathrm{KBP}$, thereby inducing its ubiquitylation and proteasomal degradation. This limits mitochondrial biogenesis. Lower panel: in differentiating cells, acetylation of KBP and expression of FBXO15 are reduced, resulting in KBP stabilization and enhanced mitochondrial biogenesis

reduction of $\mathrm{FBXO15}$ and $\mathrm{TDH}$ during early differentiation correlated with increased KBP levels. Moreover, failure to accumulate KBP levels by ectopic expression of FBX015 or deletion of $K B P$ reduced mitochondrial mass and cellular respiration, and impaired differentiation. Hence, accumulation of $\mathrm{KBP}$ is necessary to ensure mitochondrial biogenesis for faithful differentiation.

Taken together, the results by Donato et al. identify the microtubule cytoskeleton protein KBP as a key regulator of mitochondrial biogenesis in stem cells and uncover a novel ubiquitin-dependent pathway that ensures faithful stem cell proliferation and differentiation (Figure 1). At the heart of this regulatory pathway is the ubiquitin $\mathrm{E} 3$ ligase $\mathrm{SCF}^{\mathrm{Fbox} 15}$, which mediates KBP proteolysis in a manner that requires substrate acetylation by the mitochondrial enzymes GCN5L1 and TDH. These findings raise intriguing questions and open novel avenues for future investigation. How does KBP regulate the microtubule cytoskeleton to allow the growth of mitochondria? KBP binds to the kinesin KIF1Balpha and increases microtubule-mitochondria interactions and mitochondrial biogenesis, suggesting that KBP-KIF1Balpha complexes might connect mitochondria to a microtubule platform that allows for mitochondrial growth. In light of findings that KBP is an inhibitor of kinesin motor activity, ${ }^{10}$ determination of the molecular mechanism by which KBP-KIF1Balpha regulates mitochondrial biogenesis will be an interesting avenue for future research. Moreover, the degradation of KBP by $\mathrm{SCF}^{\mathrm{Fbx015}}$ relied on its prior acetylation, a process that required mitochondrial acetyl-coA produced by $\mathrm{TDH}$ and the acetyltransferase GCN5L1. This implies that SCF ${ }^{\text {Fbxo15 }}$ dependent KBP degradation could be a metabolic sensing mechanism to couple mitochondrial acetyl-coA production to mitochondrial biogenesis. It is intriguing to speculate that changes in the pool of acetyl-coA during differentiation could act in concert with the transcriptional downregulation of FBXO15 and TDH to induce mitochondrial growth during differentiation. Finally, F-box proteins frequently use the same binding mode to recognize more than one target. ${ }^{5}$ Searching for other acetylated proteins containing the FBXO15 degron motif of KBP might be a useful approach to identify novel substrates of this stem cell-specific ubiquitin ligase. Studying those substrates could reveal additional roles of $\mathrm{SCF}^{\mathrm{FBXO} 15}$ or of protein acetylation in different aspects of stem cell biology.

\section{Conflict of Interest}

MR is cofounder and consultant to Nurix, a company working in the ubiquitin space. WA declares no conflict of interest.

\footnotetext{
1. Werner A et al. Trends Cell Biol 2017, 27: 568-579.

2. Xu X et al. Cell Metab 2013; 18: 325-332.

3. Donato $\mathrm{V}$ et al. Nat Cell Biol 2017; 19: 341-351.

4. Takahashi K, Yamanaka S. Cell 2006; 126: 663-676.

5. Skaar JR et al. Nat Rev Mol Cell Biol 2013; 14: 369-381.

6. Wang J et al. Science 2009; 325: 435-439.

7. Karbowski M et al. J Cell Sci 2001; 114: 281-291.

8. Hirokawa N et al. Neuron 2010; 68: 610-638.

9. Tokuzawa $Y$ et al. Mol Cell Biol 2003; 23: 2699-2708.

10. Kevenaar JT et al. Curr Biol CB 2016; 26: 849-861.
} 